\title{
Ciclo de Mejora Docente en la asignatura de Psicología de la Atención de la Percepción del Grado en Psicología
}

\author{
JuAn PEDRo VARGas \\ Dpto. Psicología Experimental \\ Universidad de Sevilla \\ vargas@us.es \\ ORCID: https://orcid.org/0000-0002-4358-5737 \\ D.O.I.: http://dx.doi.org/10.12795/JDU.2018.i01.100 \\ Pp.: $1773-1790$
}

\section{Resumen}

La presente comunicación describe una experiencia de diseño y realización de un Ciclo de Mejora Docente de ocho horas de duración para la asignatura de Psicología de la Atención y de la Percepción del Grado en Psicología de la Universidad de Sevilla durante el curso 2018/2019. En concreto la actividad se va a aplicar al tema de Percepción del color, que reviste una gran complejidad dentro de la asignatura.

Palabras clave: Psicología de la Atención de la Percepción, Psicología, Percepción del color, docencia universitaria y experimentación docente universitaria. 


\section{Breve descripción del contexto}

La presente comunicación describe un Ciclo de Mejora Docente diseñado para adaptarlo a la asignatura Psicología de la Atención y de la Percepción del Grado en Psicología de la Universidad de Sevilla. En el grupo hay 55 alumnos de los cuales al menos el $80 \%$ asisten a clase con regularidad. La metodología con la que se ha venido impartiendo esta asignatura dependía del tipo de clase. Hasta el año pasado la asignatura se organizaba en función del número de alumnos de cada grupo. En las clases de grupo grande, a la que asistían todos los alumnos, impartíamos teoría mediante clases magistrales. En los grupos medianos asistían la mitad de los alumnos y tratábamos temas monográficos mediante seminarios guiados. En grupos pequeños, donde asistían la cuarta parte de los alumnos hacíamos experimentos y redactábamos informes. Este año, la asignatura ha cambiado su estructura y tenemos más clases de grupo grande y de grupo pequeño a cambio de la desaparición de las de grupo mediano. Esta ha sido la excusa o el motivo por el que he empezado a modificar la metodología de las clases de grupo grande para que sean más útiles. El presente ciclo de mejora se va a desarrollar en torno al último de los temas de psicología de la percepción. El tema de Percepción del color es especialmente complicado porque trata un amplio abanico de conceptos con los que los alumnos no suelen haber tenido experiencia previa. Este ciclo de mejora se desarrollará en la primera mitad de noviembre y abarcará ocho horas lectivas 


\section{Diseño previo del Ciclo de Mejora Docente}

\section{Mapa de contenidos.}

Para poder organizar de forma efectiva las tareas a realizar, se realizó un listado con los contenidos fundamentales de percepción del color clasificados en contenidos conceptuales, procedimentales y actitudinales (Porlán, 2017). Posteriormente, fueron representados en un mapa conceptual (figura 1) donde se señalan las principales relaciones que existen entre ellos.

- Características físicas asociadas al color

- Longitud de onda

- Características (diferenciales) de los colores

- Colores básicos: características

- Mezclas de colores: luces y pinturas

- Representación neural del color

- Curvas de reflectancia

- Teorías clásicas: tricromática y procesos oponentes

- Integración de la información de conos en el SNC mediante procesos oponentes

- Anomalías en la percepción del color por deficiencia en los conos

Jornadas de Formación e Innovación Docente del Profesorado | № 1 (2018) Esta obra se distribuye con la licencia Creative Commons 


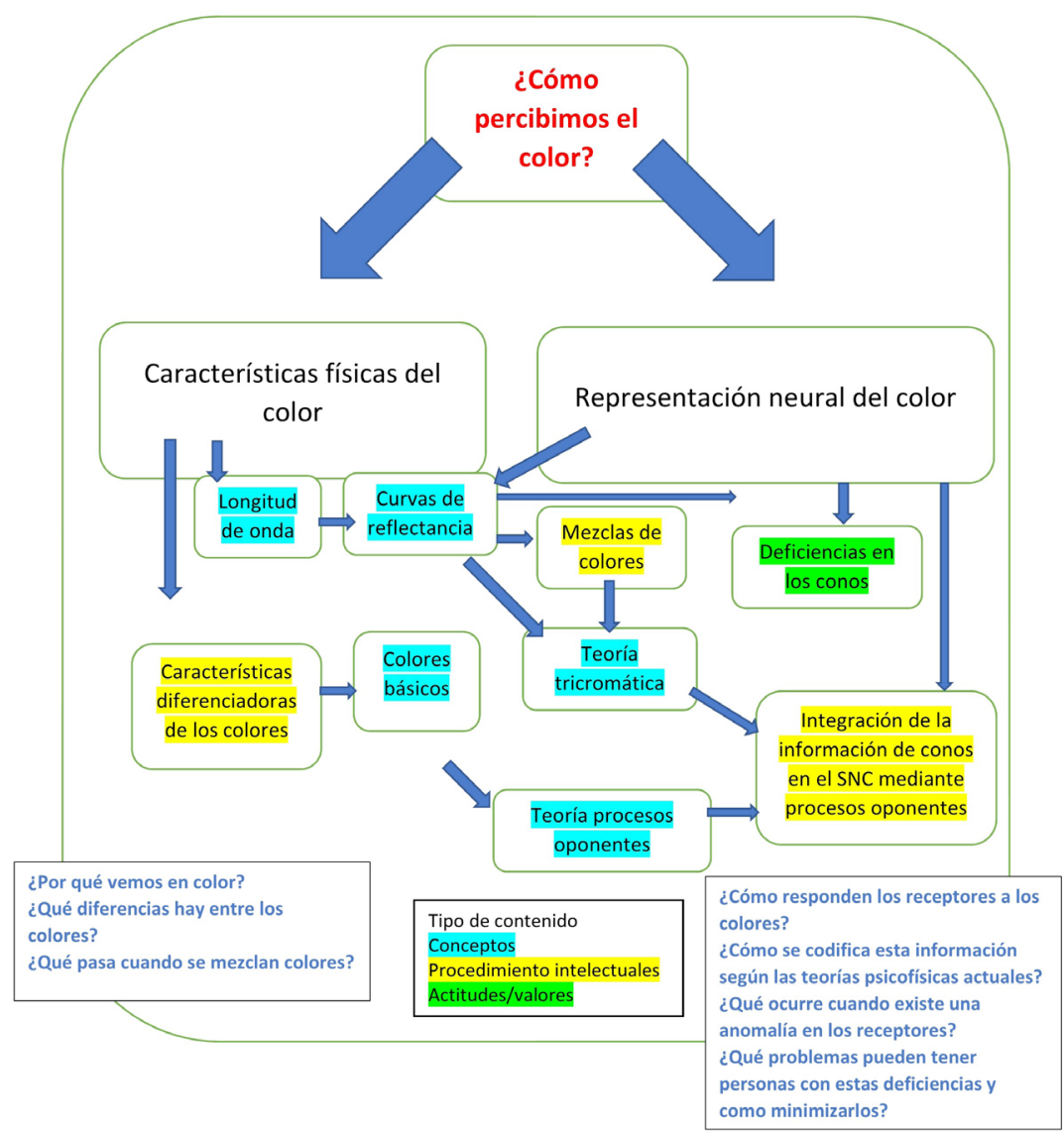

Figura 1. Cronograma

\section{Modelo metodológico}

En el modelo metodológico he intentado plasmar las actividades planificadas para las 8 horas del ciclo de mejora. Para ello he dividido en cuatro sesiones de dos horas de duración. La primera la comenzaré con la presentación del tema y del sistema de trabajo que vamos a desarrollar. El resto de la sesión los alumnos van a trabajar en grupos de 3-4 sobre las preguntas del cuestionario inicial. La segunda sesión será una puesta en común y debate sobre estas cuestiones. En la tercera empezaremos a trabajar sobre conceptos relacionados con la representación 
neural del color. Esta parte de la asignatura no estaba muy desarrollada en el cuestionario inicial porque es un tema del que no se suelen tener conceptos a priori, y que además es necesario conocer cómo se perciben las características físicas asociadas al color, que es en lo que hemos trabajado en las dos primeras sesiones. En esta sesión empezaremos con una presentación donde les expondré las teorías principales sobre percepción del color. A partir de aquí trabajaremos sobre preguntas que estaban en el cuestionario y otras preguntas más específicamente dirigidas a codificación neural. El trabajo será primero individual y luego grupal. En la cuarta sesión pondremos en común todo lo trabajado en el ciclo.

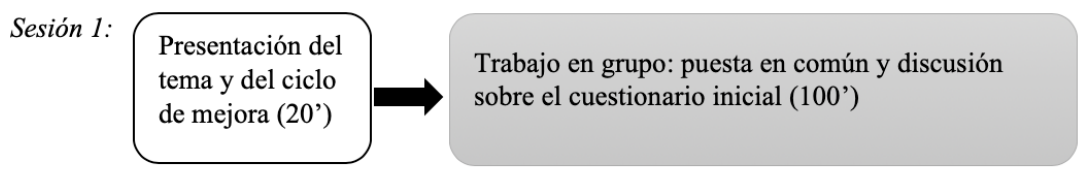

Sesión 2:

Debate y puesta en común de las respuestas al cuestionario inicial (120’)

Sesión 3:

Presentación de las principales teorías sobre percepción del color (40)
Trabajo individual sobre preguntas de codificación neural del color (40’)

Sesión 4:

Debate y puesta en común de las respuestas al cuestionario de codificación neural (80’)

Trabajo en grupo sobre preguntas de codificación neural del color (40’)
Aplicación del cuestionario inicial para evaluar el efecto de la actividad (40')

\section{Fichas de actividades}

Para organizar el trabajo durante las ocho horas de duración del ciclo de mejora, se diseñaron una serie de fichas (Porlan, 2017) donde se detalla cada una de las actividades. 
\begin{tabular}{|l|l|l|}
\hline Ficha: 1 & Fase: Sesión 1, Actividad 1 & Tiempo: 20' \\
\hline
\end{tabular}

Presentación del tema y del ciclo de mejora.

Contextualización del tema dentro de la asignatura y descripción del CMD.

Para esta primera actividad presentaré el tema de la percepción del color que es el último de los temas del bloque de psicología de la percepción. Haré hincapié en la relación que tiene que con el resto de los contenidos de la asignatura. Posteriormente les expondré el ciclo de mejora docente, que es lo que espero de ellos y cual va a ser la dinámica de trabajo en estas sesiones.

Recursos necesarios: Pizarra

\begin{tabular}{|l|l|l|}
\hline Ficha: 2 & Fase: Sesión 1, Actividad 2 & Tiempo: 100' \\
\hline
\end{tabular}

Trabajo en grupo: puesta en común y discusión sobre el cuestionario inicial En esta actividad los alumnos tienen que responder a las preguntas del cuestionario inicial, pero para ello tendrán disponible el material de la asignatura y el trabajo lo realizarán en grupos de 3-4 alumnos. Uno de los problemas que tienen los alumnos de la asignatura de psicología de la atención y de la percepción es que tienen muy claro los conceptos asociados a esta asignatura, pero son conceptos de uso cotidiano y en muchos aspectos entran en contradicción con los aspectos científicos de los mismos.

Recursos necesarios: Papel y lápiz.

\begin{tabular}{|l|l|l|}
\hline Ficha: 3 & $\begin{array}{l}\text { Fase: Sesión 2. Actividad } \\
\text { única }\end{array}$ & Tiempo: 120' \\
\hline
\end{tabular}

Debate y puesta en común de las respuestas al cuestionario inicial Se establecerá un debate para contrastar las respuestas de los diferentes grupos de trabajo e intentar llegar a un acuerdo de clase. Se solicitará a diferentes grupos, de forma voluntaria o pseudovoluntaria, que lean en voz alta sus respuestas para ir anotando en la pizarra los elementos principales de las mismas. Una vez que estén todas las características, comenzaremos un debate sobre las mismas. Esto se realizará para cada una de las preguntas de forma secuencial

Recursos necesarios: Pizarra y tiza 
Ficha: 4 Fase: Sesión 3, Actividad 1

Tiempo: 40'

Clase magistral sobre las principales teorías sobre percepción del color. Una vez que hemos discutido sobre los principales conceptos del tema, que quedaron recogidos en el cuestionario inicial, ahora trabajaremos sobre la codificación neural del color. Para ello, antes repasaremos conceptos necesarios para abordar esta fase. Durante la fase previa, puede que muchos alumnos hayan llegado ya a estos conocimientos, pero para evitar que nadie se quede atrás, incluiremos los contenidos necesarios en esta actividad. Para esta actividad es importante ir estableciendo relaciones de los que hemos discutido en clase con los conceptos que tenemos que trabajar ahora. Los alumnos pueden interrumpir en cualquier momento para preguntar cuestiones relacionadas con el tema.

Recursos necesarios: ordenador y proyector.

\begin{tabular}{|l|l|l|}
\hline Ficha: 5 & Fase: Sesión 3, Actividad 2 & Tiempo: 40' \\
\hline
\end{tabular}

Trabajo individual sobre preguntas de codificación neural del color. Para esta actividad los alumnos tendrán que trabajar las siguientes preguntas. Teniendo en cuenta la teoría tricromática y la teoría de los procesos oponentes. Propón como codificaría el sistema visual esta información a nivel neural, en cada una de sus etapas.

Propón un modelo, lo más simple posible sobre el funcionamiento del sistema de acuerdo a las teorías de percepción del color.

Recursos necesarios: Papel y lápiz.

\begin{tabular}{|l|l|l|}
\hline Ficha: 6 & Fase: Sesión 3, Actividad 3 & Tiempo: 40' \\
\hline Puesta en común y discusión en grupos sobre las preguntas de la sesión \\
anterior. \\
Se les pedirá a los alumnos que formen grupos de tres estudiantes, prefe- \\
rentemente por cuestión de cercanía. En estos grupos tienen que poner en \\
común las respuestas individuales y discutirlas para llegar a una respuesta \\
realizada por el grupo, con la idea de que sea defendida en público en la \\
siguiente sesión. \\
\hline Recursos necesarios: Papel y lápiz \\
\hline
\end{tabular}

\begin{tabular}{|l|l|l|}
\hline Ficha: 7 & Fase: Sesión 4. Actividad 1 & Tiempo: 80' \\
\hline Debate y puesta en común de las respuestas al cuestionario de codifica- \\
ción neural \\
Se establecerá un debate para contrastar las respuestas de los diferentes \\
grupos de trabajo e intentar llegar a un acuerdo de clase. Se solicitará a di- \\
ferentes grupos, de forma voluntaria o pseudovoluntaria, que lean en voz \\
alta sus respuestas para ir anotando en la pizarra los elementos principa- \\
les de las mismas. Una vez que estén todas las características, comenzare- \\
mos un debate sobre las mismas. Esto se realizará para ambas preguntas \\
de forma secuencial
\end{tabular}


Recursos necesarios: Pizarra y tiza

\begin{tabular}{|l|l|l|}
\hline Ficha: 8 & Fase: Sesión 3, Actividad 2 & Tiempo: 40' \\
\hline $\begin{array}{l}\text { Aplicación del cuestionario inicial para evaluar el efecto de la actividad } \\
\left(40^{\prime}\right)\end{array}$ \\
La última actividad de este ciclo de mejora será volver a contestar el cues- \\
tionario inicial y, de esta forma, poder medir la eficacia del CDM. Aunque \\
las preguntas habrán sido tratadas en clase de forma directa, los concep- \\
tos son lo suficientemente complejos para que sea difícil reproducir las \\
respuestas si no se ha interiorizado el contenido. \\
\hline Recursos necesarios: Papel y lápiz \\
\hline
\end{tabular}

\section{Cuestionario inicial}

El cuestionario inicial tenía como objetivo conocer las ideas previas de los alumnos respecto a este tema (Bain, 2007). Posteriormente se usará para evaluar la efectividad de la intervención (Harlan, 2017). El cuestionario fue pasado a los estudiantes antes de comenzar el ciclo de mejora. Verbalmente se informó que el cuestionario formaba parte de una actividad de innovación docente, que era anónimo y que no iba a formar parte de la calificación de la asignatura. Se les dijo que eligiesen un apodo con la idea de poder relacionar el cuestionario con el que se pasaría al final del ciclo manteniendo el anonimato. Se hizo hincapié en que no se pretendía que diesen respuestas académicas ni que usaran el material aprendido en la asignatura, sino que contestasen con el conocimiento cotidiano que tuviesen sobre las preguntas. El cuestionario se reproduce a continuación.

Percepción del color. Cuestionario inicial. Código de identificación:

El presente cuestionario forma parte de una actividad de innovación docente que pretende adecuar la materia impartida a los conocimientos iniciales de los estudiantes. Para ello tenéis que responder a las siguientes preguntas con vuestro conocimiento cotidiano, no intentando buscar una respuesta académica desde la asignatura. Responde 
a las preguntas en orden correlativo sin volver hacia preguntas previas.

1. ¿Qué importancia/ventaja tiene la percepción de los colores?

2. ¿Cuál es la diferencia física entre dos colores?

3. ¿Cómo podemos describir un color? ¿Cuáles son las características del mismo?

4. ¿Cuál es el color resultante de mezclar dos colores? Pon ejemplos, ¿por qué surgen estos colores y no otros?

5. ¿Cuáles son las deficiencias de percepción del color? ¿Por qué ocurren? ¿Qué medidas se pueden tomar para minimizar su impacto en la vida cotidiana?

\section{Aplicación del CMD}

El cuestionario de ideas previas que se realizó en la sesión previa a comenzar el ciclo de mejora ha servido para identificar los puntos débiles en los conocimientos previos de los alumnos. En la primera sesión empiezo contextualizando el tema dentro de la asignatura. La percepción del color es un tema clave en psicología de la percepción y genera bastante interés. Sin embargo, las ideas previas de los alumnos suelen estar muy alejadas del conocimiento científico de la materia. Es por ello que seleccioné este tema, haciendo un cambio en la organización de la asignatura. De esta forma, el tema de la percepción del color va a ser ahora el último de los temas del bloque de psicología de la percepción. Les hago hincapié en la relación que tiene con el resto de los contenidos de la asignatura e intento vincular el tema a lo que hemos visto previamente. Posteriormente les expongo el Ciclo de Mejora Docente, les detallo las actividades que vamos a desarrollar y que es lo que espero de ellos. En general los alumnos han estado atentos y no han intervenido mucho.

Jornadas de Formación e Innovación Docente del Profesorado I № 1 (2018) Esta obra se distribuye con la licencia Creative Commons 
Una media hora después de comenzar la clase les dije que se pusieran en grupos de tres para trabajar las preguntas del cuestionario inicial. Para ello tenían disponible el material de la asignatura. Uno de los problemas que tienen los alumnos de esta asignatura es que tienen muy claro los conceptos, pero desde un punto de vista del uso cotidiano y en muchos aspectos entran en contradicción con los aspectos científicos de los mismos. En esta etapa me ha parecido que los alumnos empiezan a organizarse mejor y aprovechan más el tiempo. Son continuas las consultas de los diferentes grupos. Cuando he visto que hay conceptos que tienen que mirar para poder responder a las preguntas he hablado en voz alta para el grupo. Algunos me pidieron salir un poco antes porque no podían seguir trabajando y querían continuar en casa. Les recordé que en la sesión siguiente íbamos a trabajar estos contenidos.

En la segunda sesión se había planteado un debate para contrastar las respuestas de los diferentes grupos a las preguntas del cuestionario inicial e intentar llegar a acuerdos de clase. Empiezo recordando la dificultad que tienen muchos conceptos de la materia, a pesar de ser conceptos de uso cotidiano. Les digo que el objetivo no es encontrar una respuesta única sino trabajar con las diferentes ideas y valorar aspectos mejorables de las mismas. Empiezo solicitando que un grupo lea en alto su respuesta a la pregunta 1. Cuando termina escribo en la pizarra los elementos principales de la misma. Sigo haciendo una ronda en la que gran parte de los grupos participan. Posteriormente establecemos un debate sobre estos elementos para matizarlo. En este apartado veo que, aunque las respuestas han mejorado sustancialmente respecto a las del cuestionario inicial, aún quedan muchos conceptos erróneos por lo que intento generar preguntas que les planteen contradicciones. Hacemos lo mismo con cada pregunta. La sesión ha sido intensa y al final se nota que hay alumnos que han bajado un poco la atención. A pesar de esto creo que la sesión ha sido muy productiva. 
Una vez que hemos trabajado las preguntas clave del tema, primero en grupos con mi supervisión y luego en debates con toda la clase, en la tercera sesión imparto una clase magistral en la que repasamos todos los contenidos de una forma más lineal. Con la retroalimentación recibida tanto de los cuestionarios de ideas previas como de las sesiones previa, me centro en los problemas que he detectado. Esto es interesante porque, aunque normalmente se le dedica más tiempo a lo que uno creo que genera mayor dificultad, en este caso, esta información es más empírica. Una vez que hemos discutido sobre los principales conceptos de psicología de la percepción, que quedaron recogidos en el cuestionario inicial, empiezo a introducirles la parte de codificación neural del color. Hasta este momento, solo hemos trabajado la parte psicofísica del tema, debido a que estos conceptos tienen que quedar claros antes de poder abordar la codificación neural. Durante la fase previa, parece que algunos alumnos han adquirido parte de estos conocimientos, pero la gran mayoría o no los tiene, o no los ha integrado con la parte psicofísica. Durante la exposición intento ir estableciendo relaciones entre lo que hemos discutido en clase y los conceptos que tenemos que trabajar ahora. Durante toda la sesión los alumnos intervienen preguntando, pues pueden interrumpir en cualquier momento, por lo que el tiempo inicialmente programado para esta actividad de 40', se alarga hasta cerca de los 60' con lo que, sobre la marcha, decido reducir el tiempo de las siguientes actividades de 40' a $30 ` c a d a$ una, para poder cuadrarlas en el horario.

A continuación, empezamos a trabajar las cuestiones de codificación neural del color de manera similar a como lo hemos hecho con la parta de psicofísica. Para ello, pongo en el proyector una diapositiva con dos preguntas y les pido que las contesten de forma individual a partir del material que han ido viendo y de los debates y explicaciones de clase. Tienen para ello unos 30 minutos. Las preguntas son las siguientes: 
1. Teniendo en cuenta la teoría tricromática y la de los procesos oponentes. Propón como codificarías el sistema visual esta información a nivel neural, en cada una de sus etapas.

2. Propón un modelo, lo más simple posible, sobre el funcionamiento del sistema de acuerdo con las teorías de percepción del color.

Durante esta actividad voy resolviendo las dudas, pero en un momento me doy cuenta de que los alumnos tienen problemas para resolver la segunda pregunta. Por ello, de nuevo sobre la marcha, decido hacer un cambio en la temporalización de las actividades, pasando a la siguiente antes de tiempo. De esta forma el trabajo individual de las preguntas quedó en 20 minutos y el trabajo grupal vuelve a tener el tiempo originalmente previsto de 40'.

Como han hecho en sesiones anteriores, les pido que formen grupos de tres estudiantes, preferentemente por cercanía. Les digo que no es obligatorio mantener los grupos de sesiones anteriores, y lo dejo a su elección. Les digo que tienen que poner en común las respuestas individuales y discutirlas para llegar a una respuesta de grupo, con la idea de que sea defendida en público en la siguiente sesión. Les indico que he detectado cierta dificultad en la segunda pregunta y les hablo un poco sobre lo que es un modelo y como se puede abordar de forma más simple. Les indico en que secciones del manual pueden mirar para orientarse.

Comenzamos la cuarta y última sesión del ciclo de mejora con una puesta en común de las respuestas a las preguntas de codificación neural. Se establece un debate para contrastar las respuestas de los diferentes grupos e intentar llegar a un acuerdo de clase. Durante la sesión queda patente que el grado de complejidad de estas preguntas es muy elevado y que, aunque tienen nociones bastante avanzadas, les cuesta integrar toda la información. Es por ello que conforme avanza la sesión mi dirección del 
debate es cada vez más patente. En ocasiones el debate se va sucediendo con continuas preguntas mías y reflexiones sobre estas preguntas.

La última parte de la sesión la dedicamos a evaluar el efecto de la actividad. Para ello la tarea fue volver a contestar el cuestionario inicial y, de esta forma, poder medir la eficacia del CDM. Aunque las preguntas habían sido tratadas en clase de forma directa, los conceptos son lo suficientemente complejos para que sea difícil reproducir las respuestas si no se ha interiorizado el contenido. Les recuerdo que tienen que poner el mismo identificador que pusieron en el cuestionario inicial e invertimos en esta tarea unos 40 minutos.

Tras completar este ciclo de mejora podemos valorar los aspectos positivos y negativos del mismo. En general creo que los positivos tienen más peso que los negativos y que estos últimos son solucionables o, al menos, minimizables para el futuro.

En primer lugar, el principal aspecto positivo de este ciclo es el hecho de haber dedicado gran cantidad de tiempo a reflexionar sobre los contenidos de la materia y a como impartirlos de forma más efectiva. Generalmente paso mucho tiempo actualizando contenidos y leyendo contenido de las asignaturas que imparto, pero no le dedico tanto a organizar los contenidos de una manera lógica. Otro aspecto positivo es el hecho de poder conocer, en tiempo real, las dificultades de los alumnos para acceder a determinado contenido. Esto ha hecho que mi labor docente se haya orientado más hacia estas cuestiones de forma más ajustada que cuando prevemos las dificultades con las que se pueden encontrar. Otro aspecto positivo es el hecho de que los alumnos desarrollan unas competencias que generalmente no se trabajan tanto en clase (debates, trabajos en grupo, defender tus ideas en público...). Como aspectos negativos está el hecho de que la materia del ciclo de mejora es muy compleja y eso dificulta el ajuste de las ideas previas de los estudiantes. Me da la 
impresión de que hay materias donde es más fácil implantar este tipo de actividades. Por otra parte, la dinámica del ciclo de mejora hace que sea necesario un mayor número de horas para impartir este tema (8 horas) para una materia que generalmente se imparte en 2 ó 3 . Este problema sería solucionable si se integra toda la materia en un ciclo y se organizan los temas para adecuarlos a este tipo de enfoque.

\section{Evaluación del CMD}

\section{Evaluación del aprendizaje de los estudiantes}

Para evaluar el CMD vamos a comparar los cuestionarios que pasamos al principio y al final. Las respuestas fueron categorizadas en función de su cercanía al conocimiento científico. A continuación, se presentan las escaleras de aprendizaje (Porlán, 2017) para cada pregunta con el porcentaje de alumnos distribuidos en los escalones, al principio (en rojo) y al final (en azul)

¿Qué importancia/ventaja tiene la percepción de los colores?

A. Imprescindible/importante/Mejora la percepción

B. Supone una ventaja adaptativa

C. Además la asocian a emociones y señales

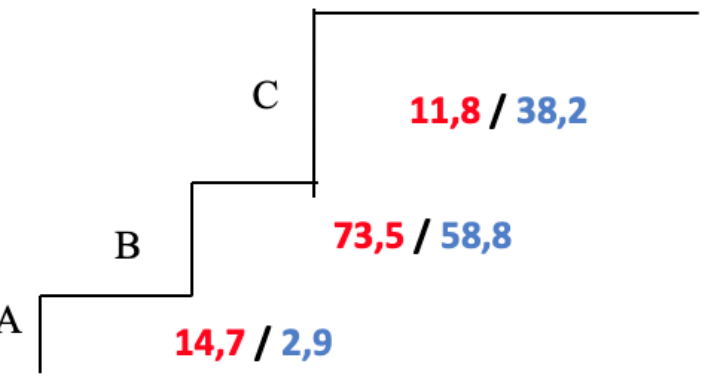

Jornadas de Formación e Innovación Docente del Profesorado | № 1 (2018)

Esta obra se distribuye con la licencia Creative Commons Reconocimiento-NoComercial-SinObraDerivada Internacional (CC BY-NC-ND 4.0.) 
¿Cuál es la diferencia física entre dos colores?
A. Lo que nos transmite
B. Tono o cantidad de luz
C. Longitud de onda
D. Curva de reflectancia

A. Pigmentos, longitud de onda, emociones

B. Distinta intensidad, comparación

C. Matiz, intensidad, brillo

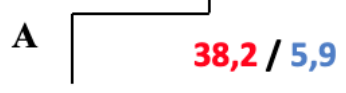

¿Cómo podemos describir un color? ¿Cuáles son las características del mismo?

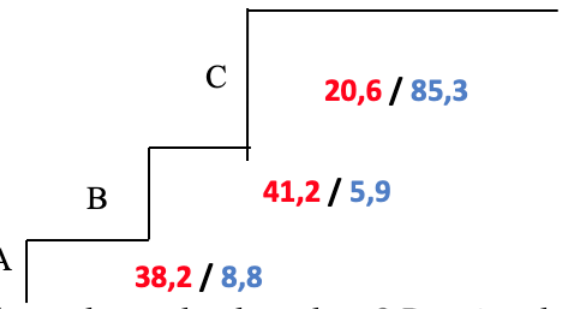

¿Cuál es el color resultante de mezclar dos colores? Pon ejemplos. ¿Por qué surgen estos colores y no otros?
A. Color secundario/color diferente
B. Mezcla de ondas
C. Diferencia de pinturas y luces
D. Sumas y restas de ondas

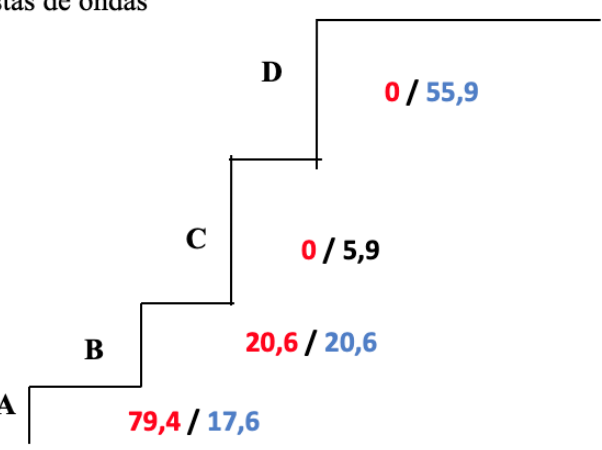

Jornadas de Formación e Innovación Docente del Profesorado | № 1 (2018) 
¿Cuáles son las deficiencias de percepción del color? ¿Por qué ocurren? ¿Qué medidas se pueden tomar para minimizar su impacto en la vida cotidiana?
A. Respuesta no relacionada
B. Daltonismo
C. Daltonismo por deficiencia en conos
D. Además con medidas adecuadas.

\section{D}

$8,8 / 32,4$

C

$23,3 / 29,4$

B

$38,2 / 35,3$

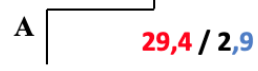

Diferencias individuales entre la prueba final y la inicial. Numero de escalones de diferencia

\begin{tabular}{|c|c|c|c|c|c|}
\hline Sujeto & Pregunta 1 & Pregunta 2 & Pregunta 3 & Pregunta 4 & Pregunta 5 \\
\hline 1 & 1 & 2 & 1 & 3 & 3 \\
\hline 2 & 0 & 2 & 0 & 0 & 1 \\
\hline 3 & 1 & 2 & 1 & -1 & 0 \\
\hline 4 & 2 & 0 & 1 & 2 & 0 \\
\hline 5 & 1 & 0 & 0 & 0 & 1 \\
\hline 6 & 0 & 2 & 2 & 1 & 0 \\
\hline 7 & 1 & 1 & -1 & 3 & 3 \\
\hline 8 & -1 & 2 & 1 & 2 & 3 \\
\hline 9 & -1 & 2 & 0 & 3 & 1 \\
\hline 10 & 0 & 0 & 2 & 0 & -1 \\
\hline 11 & 2 & 0 & 1 & 1 & 1 \\
\hline 12 & 0 & 0 & 1 & 3 & 2 \\
\hline 13 & 0 & 0 & 1 & 2 & 0 \\
\hline 14 & 0 & 3 & -1 & 3 & 1 \\
\hline 15 & 1 & 2 & 2 & 3 & 0 \\
\hline 16 & -1 & 0 & 0 & 2 & 1 \\
\hline 17 & 1 & 2 & 1 & 1 & 1 \\
\hline 18 & 0 & 2 & 2 & 1 & 1 \\
\hline 19 & 0 & 0 & 1 & 2 & -1 \\
\hline 20 & 1 & 1 & 2 & 3 & 0 \\
\hline
\end{tabular}

Jornadas de Formación e Innovación Docente del Profesorado | № 1 (2018)

(C) (7) Esta obra se distribuye con la licencia Creative Commons Reconocimiento-NoComercial-SinObraDerivada 


\begin{tabular}{c|ccccc}
21 & 0 & 0 & 2 & 0 & 3 \\
22 & 0 & 1 & 2 & 3 & 1 \\
23 & 0 & 0 & 2 & 1 & 0 \\
24 & 1 & 1 & 0 & 3 & 0 \\
25 & 1 & 1 & 1 & 2 & -1 \\
26 & -1 & 1 & -1 & 2 & 0 \\
27 & 1 & 0 & 2 & 2 & -1 \\
28 & 0 & -1 & 1 & 1 & 0 \\
29 & 1 & 2 & 2 & 3 & 2 \\
30 & 1 & 0 & 2 & 3 & 3 \\
31 & 1 & 1 & 1 & 3 & 0 \\
32 & 1 & 2 & 1 & 0 & -1 \\
33 & -1 & 2 & 0 & 3 & 3 \\
34 & 0 & 0 & 0 & 1 & 1 \\
Media & 0,38 & 0,97 & 0,94 & 1,8 & 0,8
\end{tabular}

Como puede observarse en los porcentajes, en todas las preguntas ha habido un avance significativo en la prueba final. Es de reseñar el avance en la pregunta dos, que puede deberse a que sobre ese contenido los alumnos no tenían formación previa. En los resultados individuales se observa que todos los alumnos mejoraron en la tarea final.

\section{Evaluación del ciclo de mejora}

La aplicación de este ciclo de mejora tiene muchos elementos positivos. El principal es el hecho de tener que tomar conciencia sobre los procesos de enseñanza y los contenidos a impartir teniendo en cuenta el punto de partida de los estudiantes (Finkel, 2008). Creo que simplemente el hecho de tener que trabajar estos aspectos ya supone una mejora de la práctica docente. Lo que me ha resultado más negativo es el hecho de introducir un cambio importante en una parte de la asignatura, lo que implica cambios de metodología docente, de trabajo de los 
estudiantes, de dinámica de clases. Estos cambios requieren de un periodo de adaptación o de aprendizaje que, por lo limitado del ciclo, no se aprovechan al máximo. Actualmente, en otras asignaturas que imparto estoy introduciendo elementos para mejorar la docencia sin la estructura de un ciclo de mejora. Y tengo la impresión de que son elementos positivos para la docencia. Ahora me encuentro en la disyuntiva de qué estrategia seguir en próximas asignaturas, si ir progresivamente incorporando elementos de mejora o si empezar desde cero y planificar toda la docencia siguiendo las directrices de este ciclo.

\section{Referencias bibliográficas.}

Bain, K. (2007): Lo que hacen los mejores profesores universitarios, Valencia, Universidad de Valencia.

Finkel, D. (2008): Dar clase con la boca cerrada, Valencia, Universidad de Valencia.

Harland, T. (2017): Enseñanza universitaria. Una guía introductoria, Madrid, Morata.

Porlán, R. (Coord.) (2017): Enseñanza Universitaria. Cómo mejorarla, Madrid, Ediciones Morata.

Jornadas de Formación e Innovación Docente del Profesorado I № 1 (2018) Esta obra se distribuye con la licencia Creative Commons 